\title{
Saïd Boujrouf et Ouidad Tebaa (dir.), Tourisme et pauvreté
}

coll. “Agence universitaire de la francophonie", éd. des Archives contemporaines, 2011, 270 pages

\section{Philippe Violier}

\section{OpenEdition \\ Journals}

Édition électronique

URL : http://journals.openedition.org/tourisme/487

DOI : 10.4000/tourisme.487

ISSN : 2492-7503

\section{Éditeur}

Éditions touristiques européennes

\section{Édition imprimée}

Date de publication : 1 décembre 2011

Pagination : 113-115

ISSN : 2109-5671

\section{Référence électronique}

Philippe Violier, « Saïd Boujrouf et Ouidad Tebaa (dir.), Tourisme et pauvreté », Mondes du Tourisme [En ligne], 4 | 2011, mis en ligne le 30 septembre 2015, consulté le 22 septembre 2020. URL : http:// journals.openedition.org/tourisme/487 ; DOI : https://doi.org/10.4000/tourisme.487

Ce document a été généré automatiquement le 22 septembre 2020.

\section{c) $(9)$}

Mondes du tourisme est mis à disposition selon les termes de la licence Creative Commons Attribution - Pas d'Utilisation Commerciale - Pas de Modification 4.0 International. 


\section{Lecture critique}

\section{Saïd Boujrouf et Ouidad Tebaa (dir.), Tourisme et pauvreté}

coll. “Agence universitaire de la francophonie", éd. des Archives

contemporaines, 2011, 270 pages

Philippe Violier

\section{RÉFÉRENCE}

Saïd Boujrouf et Ouidad Tebaa (dir.), Tourisme et pauvreté, coll. "Agence universitaire de la francophonie", éd. des Archives contemporaines, 2011.

1 Cet ouvrage collectif constitue les actes de deux colloques qui se sont tenus en 2008, l'un à l'université Cadi Ayyad de Marrakech, l'autre à l'université Van Lang d'Ho Chi Minh Ville. Les textes rassemblés présentent une grande cohérence (à l'exception d'un seul), ce qui constitue déjà une performance tant il est difficile de créer de la cohérence dans un colloque. Ils sont présentés en trois axes, après une introduction un peu rapide due à Saïd Boujrouf. Le statut de cet ouvrage doit être questionné : sur 22 communications, 7 sont présentées sans bibliographie, 5 comportent moins d'une dizaine de publications scientifiques; la plupart des auteurs semblent ignorer que la question du développement a déjà été abordée par des productions scientifiques (Isabelle Sacareau est ainsi citée une seule fois).

2 Le premier axe, intitulé "Entre tourisme et développement humain et social. Quel positionnement théorique et méthodologique ?", apparaît à la lecture le plus décevant, car il y est peu question de théorie et encore moins de méthode, au-delà d'un intéressant questionnement posé par Mimoun Hillali. Sans doute le titre est-il ambitieux dans un colloque qui, par définition, réunit des chercheurs développant des théories et mettant en œuvre des méthodes variées. Le pluriel eût mieux convenu (quels positionnements...) et une solide synthèse manque pour se hisser à la hauteur des ambitions affichées. Au-delà, l'absence de conceptualisation traverse l'ensemble de 
l'ouvrage. Des notions sont convoquées sans être questionnées: le tourisme de masse est méchant, le tourisme solidaire est gentil. Cela confine parfois à la pétition de principe et tient davantage du programme électoral que de l'analyse. Et nuit à l'ouvrage qui ne manque pas de textes intéressants, notamment d'études de cas qui montrent une situation bien plus contrastée que ne le laisse supposer quelques jugements hâtifs qui ne permettent de sortir du dilemme insoutenable : le tourisme ça ne va jamais ; quand il n'y en a pas, il en faudrait; quand il y en a, c'est trop. Comme le trop commence à quelques dizaines, ce qui est bien sûr insuffisant pour déclencher une spirale de développement, le lecteur n'entrevoit pas d'issue. De plus, l'irruption du tourisme induit des mutations qui sont toujours appréhendées de manière négative ("folklorisation"). Là aussi, la lecture de travaux fondamentaux, comme ceux de Picard sur Bali, manque à des approches souvent "fixistes" qui figent les sociétés étudiées dans un état de nature, nécessaire âge d'or, et leur dénient tout droit à la prise en main de leur avenir.

3 Les études de cas et approches pragmatiques constituent assurément des apports intéressants à la réflexion. Aomar Ibourk et Sahid Boujrouf proposent ainsi une communication fondée sur l'étude des corrélations entre tourisme et réduction de la pauvreté. Ce texte est révélateur. D'une part, les auteurs abordent ce qu'ils posent comme un problème : "Il s'agit de vérifier... si la politique de développement touristique profite à toutes les régions du Maroc". Or il s'agit là du fantasme du géographe: l'équilibre territorial, lequel n'existe dans aucun espace du monde, à propos d'aucune activité économique. D'autre part, leurs résultats montrent une forte corrélation pour les six premières destinations au Maroc. Dès lors, ils focalisent l'analyse sur Ouarzazate, la septième, où effectivement la présence du tourisme n'a pas aboli la pauvreté. Or, Ouarzazate est davantage un lieu de passage, un point de divergence des mobilités. Les touristes ne s'y posent guère, ce qui partout dans le monde n'est pas une situation favorable au développement économique. Saïd Boujrouf revient sur cette question de "l'équilibre" dans un texte consacré aux communes de montagne. Il souligne tout d'abord que la montagne n'a jamais été une préoccupation de la politique nationale et que le développement touristique profite à ceux qui dominent économiquement ou politiquement. Au passage, la nécessité de la formation est mentionnée. Mais il pose aussi un dilemme qui demande un approfondissement : soit le tourisme ne contribue pas assez à l'enrichissement et ne permet pas alors l'intégration de l'espace montagnard, soit lorsque c'est le cas, il détourne les populations et les moyens de l'agriculture. Que faire? Compte tenu des dynamiques sociales qu'il relève, les habitants vont se tourner vers le tourisme et là, comme ailleurs, l'agriculture en subira des effets, avant de se recomposer en relation avec la demande touristique. Un texte sur la Vallée de Crisul Repede en Roumanie, signé par Rodica Petrea, Luminita Filimon et Angela Marusca, aborde avec moins d'ambigüité la question du développement par le tourisme et relève avec acuité les faiblesses (absence d'organisation, faible connexion avec les sociétés touristiques proches, mauvais état des infrastructures), de cette unité territoriale de planification (outil mis en œuvre pour favoriser le développement).

Sébastien Preuil montre la diversité des voies empruntées par les villages du lac Tonle Sap, au Cambodge, et les effets dévastateurs de l'absence de régulation par l'État. Alain Maharaux articule de manière très convaincante le contexte étatique du Vietnam et les initiatives des villageois dans le nord. Hélas, il se perd en propos entendus sur le déclin de l'harmonie des paysages et les scories dues au tourisme (ah vivement un tourisme sans hôtels ni parking, sans effets sur l'espace mais qui, bien sûr, créerait du 
développement économique comme par magie!). Cependant, c'est le seul texte à aborder la question du tourisme intérieur, or il est, d'après l'auteur, plus orienté vers la modernité que vers la contemplation des villages et des activités traditionnelles. Tiens donc, le regard vers le passé serait-il une posture occidentale? D'ailleurs, toujours au Vietnam, deux textes permettent d'entrevoir des perspectives, l'un de Thi Thuy Hang, "Le tourisme communautaire, un instrument de lutte contre la pauvreté", l'autre de Vo Sang Xuan Lan, consacré aux villages de métier traditionnel. Les deux analysent les difficultés et les avancées du tourisme, sans la litanie des regrets éternels sur le temps qui passe.

5 La tonalité générale de l'ouvrage demeure enfermée dans un cadre de lecture dichotomique du monde, qui oppose le Nord colonisateur au Sud. S'il n'est pas douteux que le déplacement crée une coprésence entre des touristes relativement riches et des populations démunies, il convient de rappeler que là réside justement la possibilité de développement, "l'économie résidentielle" de Davezies. Ces flux, de plus, ne peuvent se produire en nombre sans la médiation des tour-opérateurs. Car les touristes ne sont pas des aventuriers, et heureusement ! - en effet, les aventuriers sont très peu nombreux et vivent à la dure ce qui, ensemble, ne crée rien. Les TO sont nécessaires, ils permettent à des individus issus d'une société caractérisée par le confort et le bien-être de circuler, en l'occurrence, dans les espaces pauvres du monde. Si, 80 à $90 \%$ des flux touristiques, selon les entreprises, sont des flux internes aux pays riches, c'est bien parce que cela exige moins d'énergie et de compétence que de fréquenter les destinations économiquement moins développées. D'un autre point de vue, des TO occidentaux, notamment ceux qui se positionnent sur des stratégies de bas prix, recherchent des partenaires locaux, et n'ont pas systématiquement recours à des établissements relevant de grands groupes. Ensuite, il ne faut pas confondre le coût d'un voyage et la marge réalisée. Enfin, les bénéfices nets liés à l'activité touristique sont faibles par rapport à ceux des grands groupes industriels de la pharmacie, par exemple.

Les flux touristiques internes ne sont abordés que de manière très incidente. Bref l'ouvrage n'a pas intégré le concept de société émergente, ou de tourisme de masse à l'échelle du monde. Car le différentiel entre le touriste et l'hôte peut être moins grand, et donc l'accueil plus aisé dans ce cas. De même, aucun texte n'aborde le rôle des élites locales et des pouvoirs politiques qui accaparent une part importante des richesses produites. Les principales destinations touristiques du Sud figurent également en bonne place dans les classements établis au sujet de la corruption. Les récents mouvements sociaux en Tunisie et Égypte l'ont bien montré.

7 Enfin, le tourisme peut contribuer au développement, à travers la création d'emplois. Les entreprises occidentales ne cherchent pas, en raison des coûts, à réserver tous les emplois aux expatriés. La création d'emplois directs dans le tourisme ou indirects, dans l'artisanat, ou l'agriculture, exige que l'habitant maîtrise les normes attendues. La formation apparaît dès lors comme un enjeu stratégique. Or cette thématique est à peine abordée dans les textes. 